\title{
DECOMPOSIÇÃO CATALÍTICA DA HIDRAZINA SOBRE IRÍDIO SUPORTADO EM COMPÓSITOS À BASE DE NANOFIBRAS DE CARBONO PARA PROPULSÃO ESPACIAL. TESTES EM CONDIÇÕES REAIS
}

\author{
Ricardo Vieira* e Demétrio Bastos Netto \\ Laboratório de Combustão e Propulsão, Instituto Nacional de Pesquisas Espaciais, Rodovia Presidente Dutra, km 40, 12630-000 \\ Cachoeira Paulista - SP, Brasil \\ Pierre Bernhardt, Marc-Jacques Ledoux, Cuong Pham-Huu \\ Laboratoire des Matériaux, Surfaces et Procédés pour la Catalyse, Université Louis Pasteur, 25 rue Becquerel, 67087 Strasbourg \\ Cedex 2, France
}

Recebido em 15/1/04; aceito em 27/8/04; publicado na web em 23/11/04

\begin{abstract}
CATALYTIC DECOMPOSITION OF HYDRAZINE OVER IRIDIUM SUPPORTED ON CARBON NANOFIBER COMPOSITES FOR PROPULSION IN SPACE: TESTS UNDER REAL-LIFE CONDITIONS. The aim of this work is to present the catalytic performance of iridium supported on carbon nanofibers with macroscopic shaping in a $2 \mathrm{~N}$ hydrazine microthruster placed inside a vacuum chamber in order to reproduce real-life conditions. The performances obtained are compared to those of the commercial catalyst Shell 405. The carbon-nanofiber based catalyst showed better performance than the commercial catalyst from the standpoint of activity due to its texture and its thermal conductivity.
\end{abstract}

Keywords: carbon nanofiber; hydrazine decomposition; satellite propulsion.

\section{INTRODUÇÃO}

Atualmente a maior parte dos satélites em órbita utilizam o sistema de propulsão a monopropelente líquido para operações de correção de órbita e posicionamento. O empuxo é obtido pela decomposição catalítica do monopropelente embarcado. Estes sistemas empregam micropropulsores a decomposição de hidrazina e um catalisador composto de 30 a $40 \%$ de irídio suportado em alumina. A decomposição da hidrazina conduz inicialmente à formação de nitrogênio e amoníaco, Equação (1), este último leva, por sua vez, à formação de hidrogênio e nitrogênio, Equação (2) ${ }^{1}$.

$3 \mathrm{~N}_{2} \mathrm{H}_{4} \rightarrow 4 \mathrm{NH}_{3}+\mathrm{N}_{2}$

$4 \mathrm{NH}_{3}+\mathrm{N}_{2} \mathrm{H}_{4} \rightarrow 3 \mathrm{~N}_{2}+8 \mathrm{H}_{2}$

A primeira é uma reação insensível à estrutura, ou seja, a atividade do catalisador dependerá da superfície metálica acessível. Em contrapartida, a decomposição do amoníaco é uma reação sensível à estrutura dos cristalitos, onde as partículas maiores de irídio são mais ativas nesta reação que as pequenas partículas. Porém, é esta última reação que contribui mais significativamente para o empuxo do micropropulsor, pois este é proporcional à seletividade do catalisador em hidrogênio, buscando sempre um ótimo de $40 \%$ em seletividade ${ }^{2}$.

A superfície específica do catalisador comercial é constituída principalmente de meso e microporos. Assim, um catalisador meso e macroporoso, sem perdas significativas de sua superfície específica, poderia incrementar o desempenho do micropropulsor promovendo o aumento da acessibilidade às grandes partículas metálicas.

Recentemente muitos autores têm sugerido a aplicação de nanofibras de carbono (NFC) como suporte de catalisadores ${ }^{3-5}$ devido (i) à grande interação metal/suporte causada pela presença de planos prismáticos na superfície das nanofibras, (ii) à elevada superfície específica, que oferece um melhor contato reativos/reagentes e (iii) à ausência de poros reduzindo os fenômenos de difusão, princi-

*e-mail: rvieira@lcp.inpe.br palmente em reações em fase líquida ou extremamente rápidas. Porém, o seu tamanho nanométrico pode ocasionar problemas durante o carregamento de reatores e de perda de carga. A fim de eliminar estes problemas, o grupo de Ledoux desenvolveu um método de preparação de nanofibras de carbono na forma macroscópica ${ }^{6}$. Este novo material foi aplicado com sucesso na reação de oxidação seletiva do $\mathrm{H}_{2} \mathrm{~S}$ a baixa temperatura e na reação de hidrogenação do cinamaldeído em fase líquida.

O mesmo grupo testou em laboratório o desempenho do compósito à base de nanofibras de carbono impregnado com $30 \%$ de irídio na decomposição catalítica da hidrazina em um micropiloto ${ }^{8}$. Os resultados promissores conduziram a ensaios em condições reais de utilização. Assim, este trabalho compara o desempenho do catalisador à base de nanofibras de carbono com o do catalisador comercial (33-36\% $\mathrm{Ir} / \mathrm{Al}_{2} \mathrm{O}_{3}$ ) em um micropropulsor de $2 \mathrm{~N}$ em câmara de vácuo.

\section{PARTE EXPERIMENTAL}

\section{Preparação do catalisador}

As nanofibras de carbono foram preparadas da mesma maneira descrita em trabalho publicado anteriormente ${ }^{9}$, ou seja, a partir da decomposição catalítica de uma mistura contendo etano e hidrogênio sobre partículas de níquel suportadas em feltro de grafite, o qual apresenta o papel de suporte macroscópico.

$\mathrm{O}$ feltro de grafite (Carbone Lorraine Co.) apresentando uma superfície específica menor que $1 \mathrm{~m}^{2} \mathrm{~g}^{-1}$ foi impregnado com $1 \% \mathrm{em}$ massa de níquel e colocado em um tubo de quartzo acoplado a um forno horizontal sob fluxo de hidrogênio. Após redução a $400{ }^{\circ} \mathrm{C}$ durante $2 \mathrm{~h}$, a temperatura foi elevada de 400 a $700{ }^{\circ} \mathrm{C}$ e o fluxo de hidrogênio foi trocado pela mistura reacional contendo hidrogênio e etano. $\mathrm{O}$ tempo de síntese foi fixado em $2 \mathrm{~h}$, obtendo-se assim um rendimento em nanofibras de $100 \%$ em relação ao peso inicial do feltro de grafite. O compósito à base de nanofibras de carbono suportadas em feltro de grafite, apresentando uma superfície específi- 
ca de $85 \mathrm{~m}^{2} \mathrm{~g}^{-1}$, foi cortado na forma de cilindros medindo $7 \mathrm{~mm}$ de diâmetro e $7 \mathrm{~mm}$ de altura. Estes cilindros foram impregnados com $30 \%$ em peso de irídio a partir do ácido hexacloroirídico e secos a $100{ }^{\circ} \mathrm{C}$ durante $2 \mathrm{~h}$. O catalisador foi reduzido a $500{ }^{\circ} \mathrm{C}$ por $2 \mathrm{~h}$, seguido de um tratamento térmico a $500{ }^{\circ} \mathrm{C}$ durante $24 \mathrm{~h}$. Este tratamento tem dois objetivos: primeiro, reduzir o teor de cloro na superfície do catalisador, diminuindo assim a mobilidade das partículas metálicas durante os testes de decomposição ${ }^{10}$ e segundo, aumentar o tamanho das partículas aumentando assim a seletividade do catalisador em hidrogênio. A Figura 1 mostra a influência do tratamento térmico no crescimento das partículas.
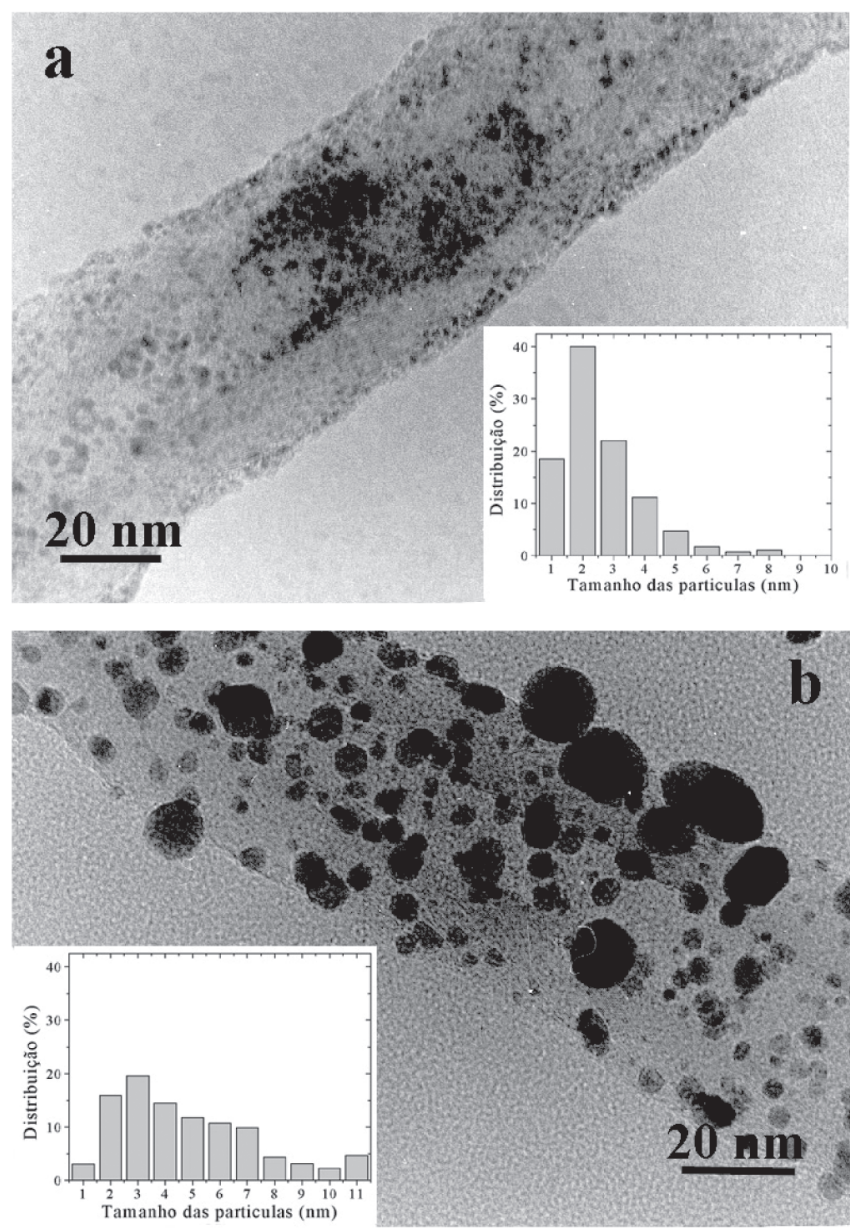

Figura 1. Imagens em Microscopia Eletrônica de Transmissão de nanofibras de carbono cobertas por partículas de irídio após redução (a) e sinterização (b) com seus respectivos histogramas de distribuição das partículas

\section{Condições dos testes}

Os catalisadores foram testados em um banco de teste de sistemas propulsivos (Figura 2) constituído de uma câmara de vácuo, que permite simular as condições de pressão no espaço, e de um sistema de aquisição de dados necessário às medidas dos principais parâmetros de desempenho dos diferentes catalisadores testados. Os ensaios de decomposição de hidrazina foram realizados em um micropropulsor de $2 \mathrm{~N}$, previsto para funcionar com o catalisador comercial.

Os testes foram realizados em dois diferentes níveis de pressão de alimentação de hidrazina, a 22 e 5.5 bar, simulando assim as reais condições de pressão do reservatório de propelente do satélite no início e no final de sua vida útil, respectivamente. Para cada nível de pressão foram realizados quatro séries de 100 tiros pulsados e 1 tiro
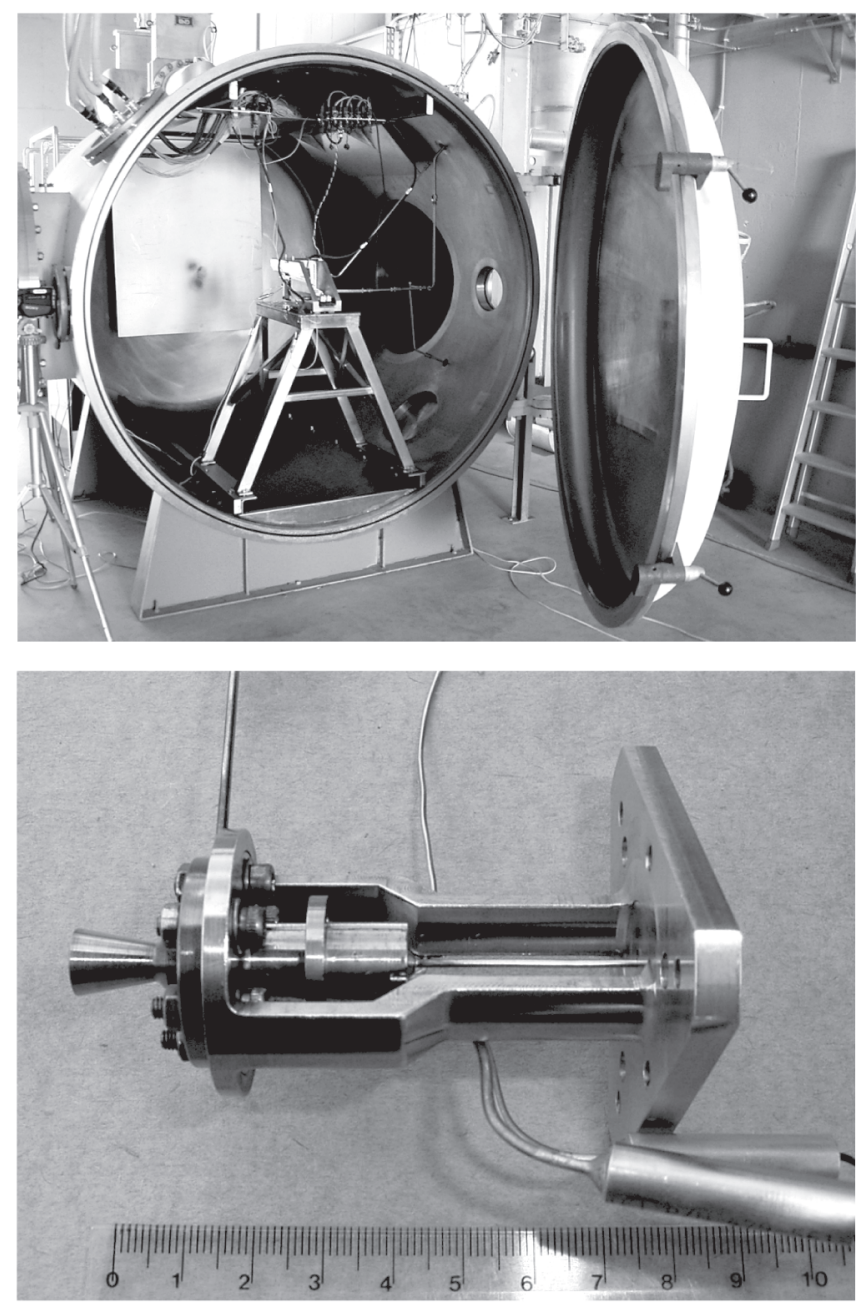

Figura 2. Fotografia da câmara de vácuo do Banco de Testes do INPE e do micropropulsor de $2 \mathrm{~N}$ utilizado nos testes

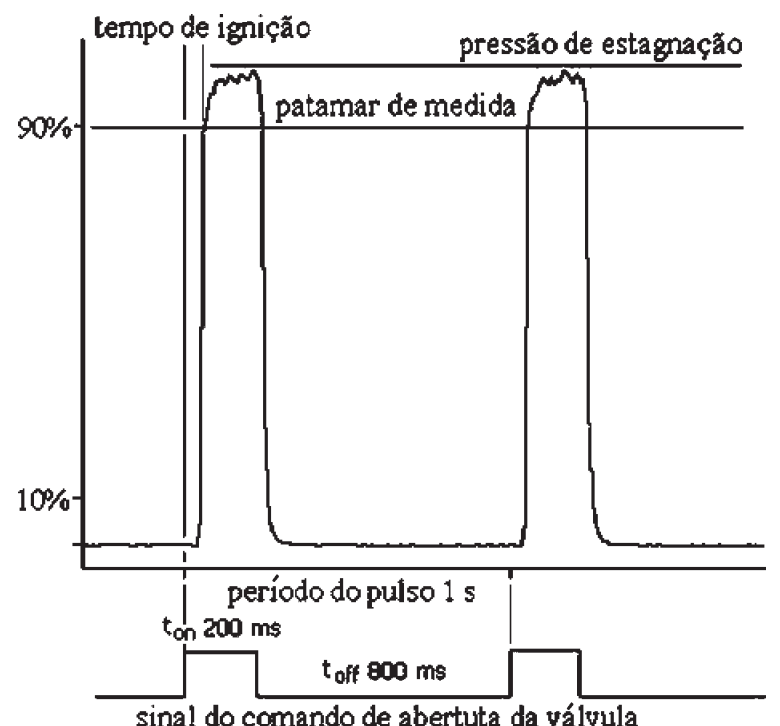

Figura 3. Gráfico representativo de um pulso de injeção de propelente de $200 \mathrm{~ms}$ em um período de $1 \mathrm{~s}$

contínuo. A Figura 3 ilustra as condições de funcionamento da eletroválvula durante os testes realizados no micropropulsor, descritos na Tabela 1. 
Tabela 1. Condições de funcionamento da eletroválvula

\begin{tabular}{cccc}
\hline Teste & $\begin{array}{c}\text { Pressão de } \\
\text { injeção do } \\
\text { propelente } \\
\text { (bar) }\end{array}$ & $\begin{array}{c}\text { Duração de } \\
\text { abertura da } \\
\text { eletroválvula }-\mathrm{t}_{\text {on }} \\
(\mathrm{ms})\end{array}$ & $\begin{array}{c}\text { Duração de } \\
\text { fechamento da } \\
\text { eletroválvula }-\mathrm{t}_{\text {off }} \\
(\mathrm{ms})\end{array}$ \\
\hline $\mathbf{1}$ & 5,5 & 20 & 980 \\
$\mathbf{2}$ & 5,5 & 100 & 900 \\
$\mathbf{3}$ & 5,5 & 200 & 800 \\
$\mathbf{4}$ & 5,5 & 500 & 500 \\
$\mathbf{5}$ & 5,5 & 5000 & - \\
$\mathbf{6}$ & 22 & 20 & 980 \\
$\mathbf{7}$ & 22 & 100 & 900 \\
$\mathbf{8}$ & 22 & 200 & 800 \\
$\mathbf{9}$ & 22 & 500 & 500 \\
$\mathbf{1 0}$ & 22 & 5000 & - \\
\hline
\end{tabular}

Os testes no micropropulsor geram um forte aumento de temperatura no leito catalítico e, conseqüentemente, variações na estrutura porosa e diminuição da superfície metálica do catalisador. Os testes em modo pulsado submetem o catalisador a grandes oscilações de pressão, permitindo também a avaliação de sua resistência mecânica.

Uma vez que todos catalisadores testados são à base de irídio e que este metal decompõe espontaneamente a hidrazina, decidiu-se iniciar todos os testes a $120^{\circ} \mathrm{C}$.

\section{Avaliação dos desempenhos}

Os parâmetros de avaliação de desempenho dos catalisadores no micropropulsor adotados foram o nível e a estabilidade do empuxo, e o tempo de ignição da reação, este último definido como o período decorrido entre a abertura da eletroválvula e o momento em que a pressão atinge $90 \%$ do valor da pressão de estagnação (ver Figura 3).

\section{RESULTADOS E DISCUSSÃO}

As dimensões da câmara de reação do micropropulsor empregado são $6,7 \mathrm{~mm}$ de diâmetro por $20 \mathrm{~mm}$ de comprimento. O micropropulsor foi carregado com $1,144 \mathrm{~g}$ do catalisador de referência e com $0,15 \mathrm{~g}$ do catalisador à base de nanofibras de carbono (NFC).

A Tabela 2 apresenta os valores médios de empuxo de séries de 100 tiros em diferentes condições de injeção de hidrazina.

$\mathrm{O}$ catalisador à base de nanofibras de carbono apresentou valores de empuxo (F) superiores aos do catalisador de referência (Figura 4). Este melhor desempenho pode ser explicado pela grande superfície metálica exposta pelo catalisador à base de nanofibras, apesar da massa introduzida no micropropulsor ser dez vezes inferior à do catalisador comercial. Cumpre ressaltar que a superfície específica das nanofibras de carbono $\left(85 \mathrm{~m}^{2} \mathrm{~g}^{-1}\right)$ é externa, ou seja, ela não é fechada em poros como no caso do catalisador comercial $\left(115 \mathrm{~m}^{2} \mathrm{~g}^{-1}\right)$. Neste tipo de reação, onde as transferências de massa e de calor devem ser muito rápidas, o reagente não tem tempo de penetrar nos poros e a reação se passa praticamente na superfície externa dos grãos do catalisador. Este fato pode ser confirmado pelo aumento de desempenho do Shell 405 em relação ao Ir/NFC nos testes a 22 bar. A elevação da pressão contribuiu para a penetração do propelente nos poros do catalisador de referência, melhorando assim os seus resultados.

Os valores de temperatura $\left(\mathrm{T}_{\mathrm{c}}\right)$ apresentados na Tabela 2 representam os valores máximos medidos na parede externa do micropropulsor durante cada ensaio de 100 tiros consecutivos. Os valores mais baixos apresentados pelo catalisador Ir/NFC em relação ao catalisador de referência são devidos ao fato de que a
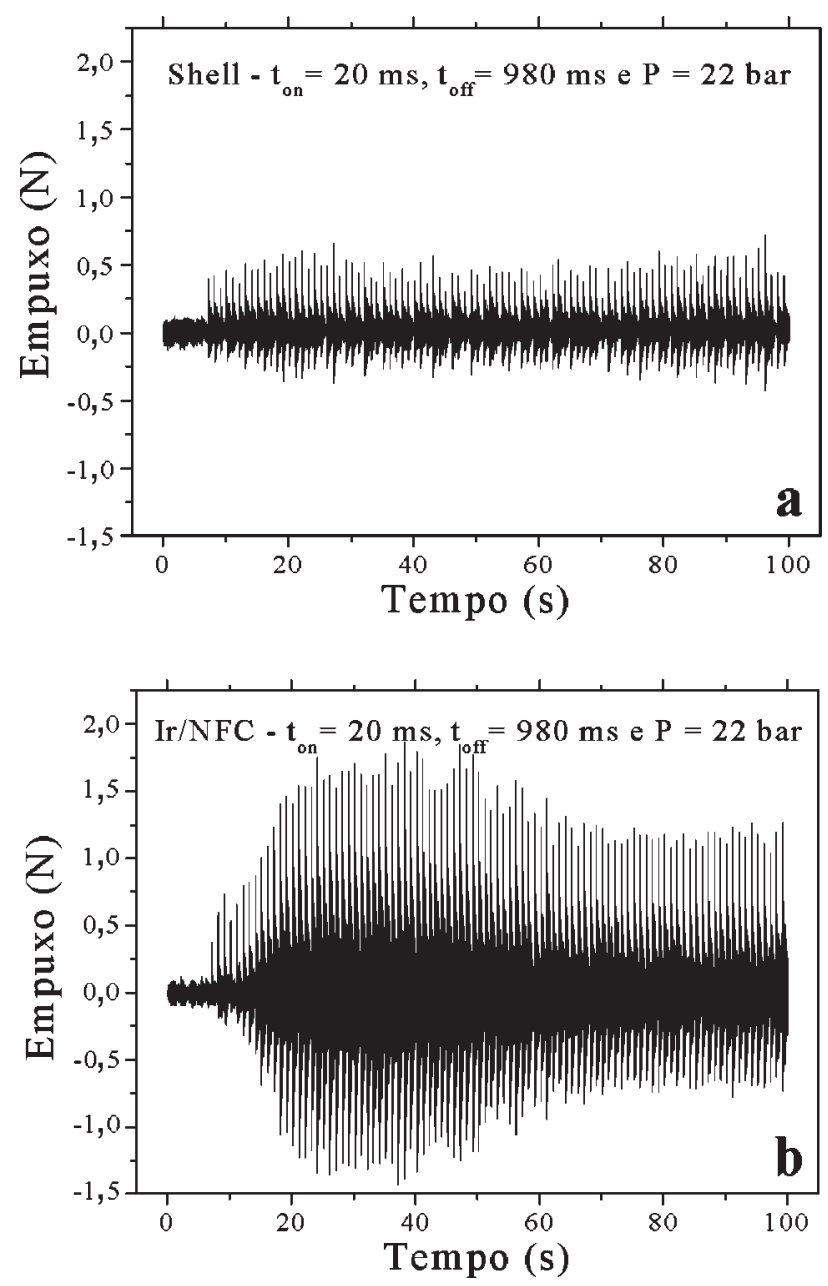

Figura 4. Comparação dos desempenhos dos catalisadores Shell $405 e$ Ir/NFC a partir da curva de empuxo de uma série de 100 tiros pulsados de 20 min e período de $1 \mathrm{~s}$

Tabela 2. Valores médios do empuxo $(\mathrm{F})$ em $\mathrm{N}$ e da temperatura da câmara de reação $\left(\mathrm{T}_{\mathrm{c}}\right)$ em ${ }^{\circ} \mathrm{C}$ a partir de uma série de 100 tiros realizados em dois diferentes níveis de pressão e em ciclos onde $t_{\text {on }}$ é a duração de abertura da eletroválvula e $t_{\text {off }}$ a de fechamento

\begin{tabular}{|c|c|c|c|c|c|c|c|c|c|}
\hline & \multirow{2}{*}{$\begin{array}{l}\text { Pressão de Injeção } \\
\text { (bar) }\end{array}$} & \multicolumn{2}{|c|}{$\mathrm{t}_{\text {on }} 20-\mathrm{t}_{\text {off }} 980$} & \multicolumn{2}{|c|}{$\mathrm{t}_{\text {on }} 200-\mathrm{t}_{\text {off }} 800$} & \multicolumn{2}{|c|}{$\mathrm{t}_{\text {on }} 500-\mathrm{t}_{\text {off }} 500$} & \multicolumn{2}{|c|}{$\mathrm{t}_{\text {on }} 5000$} \\
\hline & & $\mathbf{F}$ & $\mathbf{T}_{\mathrm{c}}$ & $\mathbf{F}$ & $\mathbf{T}_{\mathrm{c}}$ & $\mathbf{F}$ & $\mathbf{T}_{\mathrm{c}}$ & $\mathbf{F}$ & $\mathbf{T}_{\mathrm{c}}$ \\
\hline Shell 405 & 5,5 & 0,34 & 380 & 0,63 & 610 & 0,66 & 680 & 0,44 & 370 \\
\hline Ir/NFC & 5,5 & 0,40 & 340 & 0,85 & 610 & 0,75 & 660 & 0,45 & 250 \\
\hline Shell 405 & 22 & 0,44 & 440 & 2,25 & 730 & 2,25 & 780 & 1,40 & 480 \\
\hline Ir/NFC & 22 & 1,20 & 420 & 2,65 & 560 & 2,20 & 640 & 1,40 & 350 \\
\hline
\end{tabular}


condutividade térmica do carbono é mais elevada que a da alumina, o que leva a uma melhor evacuação do calor gerado pela reação.
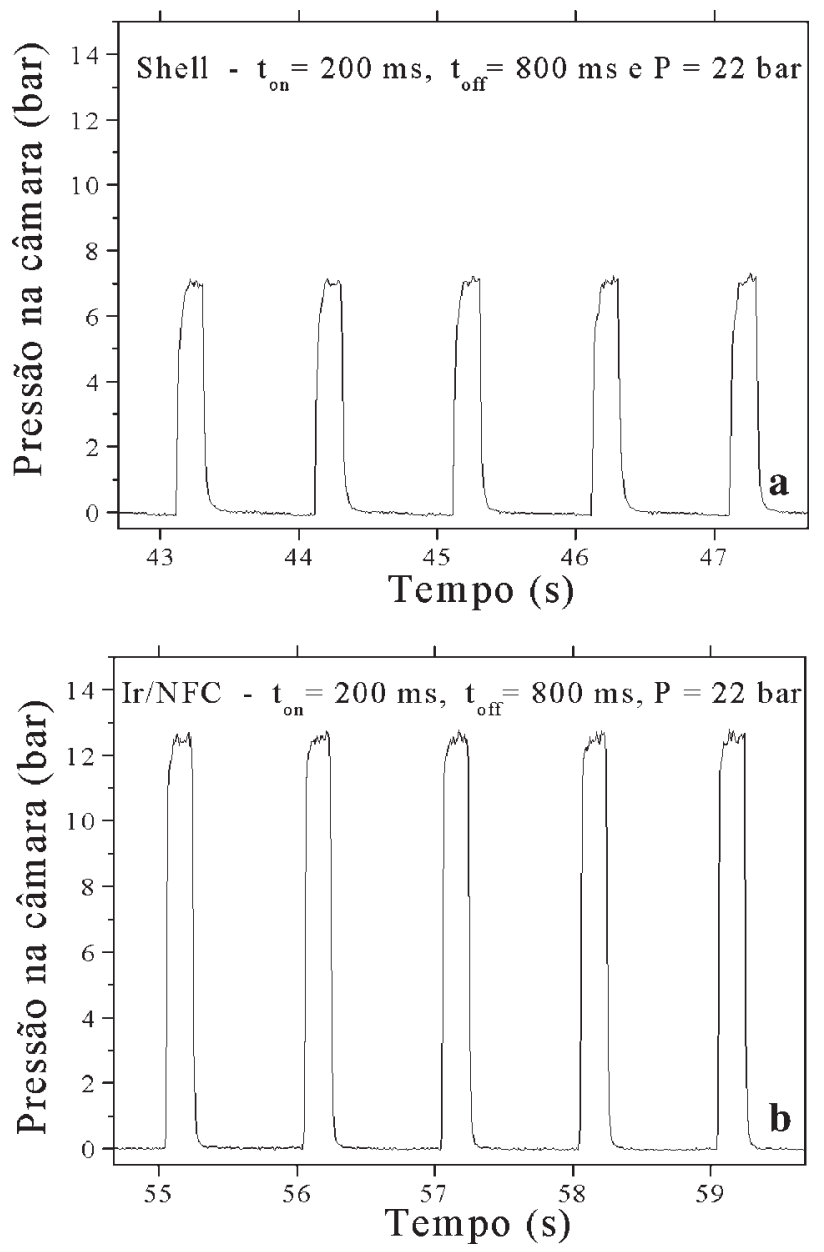

Figura 5. Curvas de pressão da câmara de reação de uma parte da série de 100 tiros pulsados de 200 min e período de 1 s dos catalisadores Shell 405 e $\operatorname{Ir} / \mathrm{NFC}$

As curvas de pressão mostram a evolução da pressão na câmara de reação, gerada pela decomposição de uma quantidade conhecida de hidrazina. Elas têm como objetivo a verificação da estabilidade dos catalisadores na decomposição do propelente. Os principais sinais de falha do catalisador são instabilidade de pressão superior a $10 \%$ do valor da pressão de estagnação e elevações súbitas de pressão ("spikings") superiores a 50\% deste mesmo valor ${ }^{11}$. Todavia, não foram observadas tais falhas nos testes dos referidos catalisadores no micropropulsor.

O tempo de ignição da reação apresentado pelos catalisadores foi medido com o auxílio de um programa de computador desenvolvido no LCP/INPE. Para tanto, foi considerado o tempo decorrido após o acionamento da eletroválvula até a pressão atingir $90 \%$ do valor da pressão de estagnação, isto para cada um dos 400 pulsos efetuados em cada nível de pressão. Os resultados confirmaram a maior eficiência do catalisador à base de nanofibras de carbono no nível de pressão mais baixo, ou seja, o catalisador Ir/NFC apresentou um tempo de ignição médio de $46 \mathrm{~min}$ a 5,5 bar enquanto o catalisador Shell foi de $62 \mathrm{~min}$. Nos testes a 22 bar os catalisadores apresentaram desempenhos similares, o tempo de ignição médio do catalisador Ir/NFC foi de $41 \mathrm{~min}$ e o do Shell de $43 \mathrm{~min}$, porém o catalisador Ir/NFC ainda é mais espontâneo na reação de decomposição da hidrazina mesmo com a elevação da pressão de injeção do propelente. O aumento do desempenho do catalisador Shell no nível mais alto de pressão de injeção deve-se ao fato de que este colabora para a penetração do propelente nos poros do catalisador, permitindo aos sítios ativos localizados no interior dos poros participarem da reação.

\section{CONCLUSÃO}

Os resultados obtidos mostram que o catalisador à base de irídio depositado sobre um suporte macroscópico constituído de nanofibras de carbono apresentou melhor desempenho na reação de decomposição da hidrazina que o seu homólogo suportado em alumina. Este melhor desempenho foi atribuído principalmente a dois fatores: primeiro, à grande superfície externa e à morfologia das nanofibras de carbono, que facilitam a difusão do reagente no catalisador; segundo, à alta condutividade térmica do suporte em carbono, que permite uma rápida homogeneização do calor gerado pela reação evitando, assim, a formação de pontos quentes nefastos à boa dispersão da fase ativa.

O desempenho do catalisador comercial aumentou com a elevação da pressão de injeção do propelente, a qual propicia maior penetração da hidrazina nos poros do catalisador e o acesso aos sítios ativos localizados no interior dos poros.

Finalmente, o suporte macroscópico à base de nanofibras de carbono pode também ser utilizado eficazmente como suporte catalítico em reações de fase líquida, onde os fenômenos de difusão dos reagentes são predominantes, e em reações que apresentam alta transferência de massa e calor.

\section{REFERÊNCIAS}

1. Schmidt, E. W.; Hydrazine and its derivatives. Preparation, Properties and Applications, John Wiley: New York, 1984.

2. Balcon, S.; Tese de Doutorado, Université de Poitiers, France, 1996.

3. Rodriguez, N. M.; Kim, M. S.; Baker, R. T. K.; J. Phys. Chem. 1994, 98, 108.

4. Salman, F.; Park, C.; Baker, R. T. K.; Catal. Today 1999, 53, 385.

5. Pham-Huu, C.; Keller, N.; Charbonniére, R.; Ziessel, R.; Ledoux, M. J.; Chem. Commun. 2000, 1871.

6. Pham-Huu, C.; Vieira, R.; Charbonnière, L.; Ziessel, R.; Ledoux, M. J.; French pat. 01151782001.

7. Nhut, J. M.; Vieira, R.; Pesant, L.; Tessonnier, J. P.; Keller, N.; Ehret, G.; Pham-Huu, C.; Ledoux, M. J.; Catal. Today 2002, 76, 11,.

8. Vieira, R.; Pham-Huu, C.; Keller, N.; Ledoux, M. J.; Chem. Commun. 2002, 954.

9. Vieira, R.; Pham-Huu, C.; Keller, N.; Ledoux, M. J.; Quim. Nova 2003, 26,665 .

10. Balcon, S.; Mary, S.; Kappenstein, C.; Gengembre, E.; Appl. Catal. 2000, 196, 179.

11. Sayer, C. F.; Induction Period of the Decomposition of Hydrazine on the Shell 405 Catalysts, TR-003808/36A, USA, 1975. 